\title{
HRJ
}

v.2 n.9 (2021)

Recebido: $11 / 11 / 2020$

Aceito: $22 / 12 / 2020$

\section{Contribuição dos programas de residência atuantes na atenção primária à saúde frente à COVID-19}

\author{
Síntique Priscila Alves Lopes ${ }^{1}$ \\ Michele Alcântara de Almeida ${ }^{2}$ \\ Thaís Aparecida de França Rocha ${ }^{3}$ \\ Maria Cristina Teixeira Cangussu ${ }^{4}$
}

${ }^{1}$ Cirurgiã-dentista especialista em Saúde da Família, mestranda do Programa de Pós-Graduação em Odontologia e Saúde vinculado à Universidade Federal da Bahia (UFBA) e preceptora do Programa de Residência Multiprofissional em Saúde da Família pela Fundação Estatal Saúde da Família - FESFSUS/Fiocruz-Ba.

${ }^{2}$ Enfermeira residente do Programa de Residência Multiprofissional em Saúde da Família pela Fundação Estatal Saúde da Família - FESF-SUS/Fiocruz-Ba.

${ }^{3}$ Cirurgiã-dentista especialista em Saúde da Família pela Fundação Estatal Saúde da Família - FESFSUS/Fiocruz-Ba.

${ }^{4}$ Cirurgiã-dentista mestre em Saúde Coletiva e doutora em Saúde Pública pela Universidade de São Paulo (USP). Atualmente docente associada da Faculdade de Odontologia da Universidade Federal da Bahia (UFBA) vinculada ao Programa de Pós-Graduação em Odontologia e Saúde

E-mail para correspondência: alvessintique@gmail.com

Sem conflito de interesse

\section{RESUMO}

Introdução: A Doença Coronavírus 2019 - COVID-19 provocou alerta global. No Brasil, os Programas de Residência tiveram sua potencialidade reconhecida pelo governo federal por meio de incentivo financeiro através do Programa "O Brasil conta comigo - Residentes na área da saúde". Objetivo: Realizar uma revisão integrativa da literatura voltada às experiências de profissionais integrantes de Programas de Residência Multiprofissional e Médica atuantes na Atenção Primária no contexto da pandemia da COVID-19 entre março e outubro de 2020. Metodologia: A busca foi realizada através da análise retrospectiva de normativas bem como por meio das bases de dados eletrônicas Pubmed/MEDLINE, Scielo, LILACS, Google Acadêmico, BVS, BDTD e CINAHL. Resultados e Discussão: Foram identificados cinco artigos cujo elo comum foram ações dos residentes pautadas no acolhimento de sintomáticos respiratórios e utilização da tecnologia para aprimoramento do cuidado à população geral. Considerações finais: Observou-se número reduzido de publicações que envolvessem o fazer destes profissionais no período referido. Fato controverso ao esperado em decorrência ao estímulo financeiro do governo federal para o enfrentamento do vírus.

Palavras-chave: Atenção Primária à Saúde, Infecções por Coronavírus, Equipe Multiprofissional, Educação Pós-Graduação, Internato e Residência. 


\title{
Contribution of residency programs acting in primary care in front of COVID-19
}

\begin{abstract}
Introduction: Coronavirus 2019 Disease - COVID-19 has caused global alert. In Brazil, the Residency Programs had their potentiality recognized by the federal government through financial incentives through the Program "Brazil counts on me - Residents in the health area". Objective: To carry out an integrative review of the literature focused on the experiences of professionals in Multiprofessional and Medical Residency Programs working in Primary Care in the context of the COVID-19 pandemic between March and October 2020. Methodology: The search was carried out through the retrospective analysis of norms as well as through the electronic databases Pubmed/MEDLINE, Scielo, LILACS, Google Academic, VHL, BDTD and CINAHL. Results and Discussion: Seven articles were identified whose common link was the actions of the residents based on the reception of respiratory symptoms and the use of technology to improve the care of the general population. Final Considerations: A reduced number of publications involving these professionals were observed in the referred period. Controversial fact as expected due to the financial stimulus of the federal government to face the virus.
\end{abstract}

Keywords: Primary Health Care, Coronavirus Infections, Multiprofessional Team, Postgraduate Education, Internship and Residence.

\section{INTRODUÇÃO}

Em 31 de dezembro de 2019, a Organização Mundial da Saúde (OMS) foi alertada sobre vários casos de pneumonia na cidade de Wuhan, província de Hubei, na República Popular da China. Tratava-se de uma nova cepa de coronavírus que não havia sido identificada antes em seres humanos ${ }^{1 \cdot}$ A doença COVID-19 (Coronavirus Disease 2019) é uma infecção, a princípio respiratória, provocada pelo vírus causador da Síndrome Respiratória Aguda Grave 2 (SARS$\mathrm{CoV}-2)^{2}$

Em 11 de março de 2020, a doença foi caracterizada como pandemia tendo em vista altas taxas de contágio e disseminação do vírus em vários países e regiões do mundo ${ }^{3}$. No Brasil, o primeiro caso foi registrado em 26 de fevereiro de 2020 pelo Ministério da Saúde após a confirmação da doença em um homem de 61 anos residente no estado de São Paulo o qual retornara de uma viagem à Itália ${ }^{4}$. 
No Brasil, o cenário da pandemia tem sido desastroso, alcançando elevado número de óbitos. Até a data da redação deste artigo, em vinte de outubro de 2020 foram confirmados 5.235.344 casos da doença e 153.905 mortes segundo o boletim diário da $\mathrm{OMS}^{5}$. Além disso, o país deparou-se inicialmente com a ausência da implantação de ações e estratégias eficazes de controle da disseminação assim como orientações inicialmente contraditórias entre as esferas de governo ${ }^{6}$.

Assim, para nortear os profissionais de saúde no enfrentamento da COVID-19, o Ministério da Saúde produziu cartilhas e manuais com orientações e recomendações sobre a organização de fluxos assistenciais e de processo de trabalho na Atenção Primária a Saúde e atuação das equipes de Saúde da Família para os atendimentos de pacientes sintomáticos respiratórios $^{7,8,9}$. Por isso, é fundamental que todos os profissionais atuantes neste nível de atenção tenham acesso aos materiais atualizados (protocolos, procedimento operacional padrão e notas técnicas), bem como saibam os fluxos de referências para pacientes graves ${ }^{10}$.

Neste sentido, observou-se que o governo federal reconheceu a importância dos Programas de Residência no enfrentamento à COVID-19 a partir da criação do programa “O Brasil conta comigo - Residentes na área da saúde" estabelecido pela Portaria n ${ }^{\circ} 580$ de 27 de março de 2020. Esta portaria objetivou ampliar a cobertura na assistência em todos os níveis de atenção e reduzir o tempo de espera nos atendimentos de usuários do SUS. Através deste programa houve um incentivo financeiro adicional no valor de 667,00 reais para os profissionais de saúde que cursam Programas de Residência Médica e Residência em Área Profissional da Saúde ${ }^{11}$.

A Residência em Área Profissional da Saúde foi criada em 2005, a partir da promulgação da Lei ${ }^{\circ}$ 11.129. Definida como modalidade de ensino de pós-graduação Lato Sensu, é voltada para a educação em serviço e destinada às categorias profissionais que integram a área de saúde, excetuada a médica ${ }^{12}$. No tocante a área médica voltada para a APS, 
a Portaria 1.143 de 2005 do Ministério da Saúde, foi instituída como forma de apoiar os programas de residência médica em Medicina de Família e Comunidade, mediante o provimento de bolsas para a Educação pelo Trabalho ${ }^{13}$. Já a Lei 12.871 de 2013, foi publicada para ampliar a formação de especialistas em Medicina de Família e Comunidade, instituindo o Programa Mais Médicos para o Brasil (PMMB). Ademais, a lei determinava que, até o final de 2018, houvesse o mesmo número de vagas de residência de acesso direto que o número de egressos dos cursos de graduação do ano anterior ${ }^{14}$.

Rosa e Vasconcelos ${ }^{15}$ (2014) ressaltaram a importância de programas de residência voltados à Atenção Primária, tendo em vista a restrição da formação acadêmica para a realidade neste nível de atenção. Os programas de residência trazem novas possibilidades aos ingressantes por meio de um campo prático (trabalho) e reflexivo (acadêmico) numa perspectiva de qualificação adequada para atender as necessidades do sistema de saúde ${ }^{16}$. Além disso, podem ser compreendidos como uma oportunidade de aprendizado e contato com profissionais de diferentes áreas, permitindo que os residentes assumam uma nova conduta na prática profissional onde a assistência aos usuários adquire um caráter mais humanizado e abrangente ${ }^{17}$.

Portanto, após explanação de aspectos relevantes quanto à atuação dos programas de residência, este trabalho tem o objetivo de realizar uma revisão integrativa da literatura sobre as experiências desenvolvidas na Atenção Primária a Saúde por profissionais integrantes de Programas de Residência Multiprofissional e Médica no contexto da pandemia da COVID-19 entre março e outubro de 2020.

\section{METODOLOGIA}

Este estudo trata-se de uma revisão integrativa da literatura cuja proposta envolve a compreensão abrangente de um determinado tema e aponta lacunas do conhecimento que 
necessitam ser exploradas. Para tanto, fomenta o seguinte rigor para a realização: identificação do problema, pesquisa bibliográfica, análise dos dados e apresentação destes ${ }^{18}$.

Neste sentido, de forma preliminar à identificação do problema, uma questão norteadora foi estabelecida: Durante a pandemia causada pelo SARS-Cov-2 no Brasil, quais seriam as ações de enfrentamento à Covid-19 estabelecidas pelos Programas de Residência Médica e Multiprofissional que atuam na Atenção Primária à Saúde? Para respondê-las, realizou-se pesquisa bibliográfica nas seguintes bases de dados: Google Acadêmico, PubMed/MEDLINE, SciELO - Scientific Eletronic Library Online, LILACS - Literatura Latino-Americana e do Caribe em Ciências da Saúde, BVS - Biblioteca Virtual de Saúde, BDTD - Biblioteca Digital Brasileira de Teses e Dissertações e CINAHL- Cumulative Index to Nursing and Allied Health Literature.

Para tanto, foram utilizados os seguintes descritores DeCS/MeSH e palavras-chave nas línguas portuguesa, inglesa e espanhola: Primary Health Care, Coronavirus Infections, Patient Care Team, Education, Graduate, Internship and Residency. Com os operadores booleanos foram realizadas as seguintes associações: Primary Health Care and Cornavirus Infections and Education, Graduate. Primary Health Care and Internship and Residency and Cornavirus Infections.

Quanto aos critérios de inclusão foram estabelecidos: artigos de pesquisa original e relato de experiências, monografias, dissertações e teses publicados em inglês, espanhol ou português que retratassem ações ou adaptações ao processo de trabalho para enfrentamento à Covid-19 estabelecidas por Programas de Residência Médica e Multiprofissional publicados entre março e outubro de 2020. No tange aos critérios de exclusão, considerou-se: estudos com desenho de pesquisa ou objetivos pouco definidos ou pouco explicitados, editoriais, matérias jornalísticas, de análise conjuntural, avaliação de protocolos, discussão teórica de conceitos, artigos não originais (como resenhas, comentários) e revisão bibliográfica. Ademais, foram 
excluídos da amostra, artigos com relatos de experiência sobre atenção básica sem residência instituída neste espaço, bem como, trabalhos que traziam a residência multiprofissional, entretanto em âmbito hospitalar. Para completude da pesquisa, referências empregadas pelos autores dos artigos foram utilizadas, não sendo realizada pesquisa manual.

A recolha e análise dos dados foi realizada entre os meses de setembro, outubro e novembro de 2020, por dois pesquisadores separadamente. Quanto aos resultados, estes foram comparados e as discordâncias resolvidas por consenso. Para a seleção das publicações incluídas no estudo foi utilizado o diagrama PRISMA ${ }^{19}$, representado na Figura 1.

Em síntese, após a identificação do problema, realizou-se pesquisa bibliográfica que evidenciou número reduzido de publicações sobre o tema proposto. Posteriormente prosseguiuse a interpretação dos dados, análise destes e apresentação estruturada. Na etapa inicial foram identificados os problemas ou as questões norteadoras do estudo, em seguida, definiu-se as bases de dados para as buscas. Por conseguinte, os dados foram ordenados e categorizados com o auxílio de um quadro teórico, onde foram registradas as informações sobre os estudos. Finalmente, realizou-se a apresentação dos resultados, com a síntese de elementos importantes em uma soma integradora do assunto conforme Quadro 1.

Figura 1. Fluxograma atinente ao processo de seleção dos estudos para a revisão integrativa da literatura.
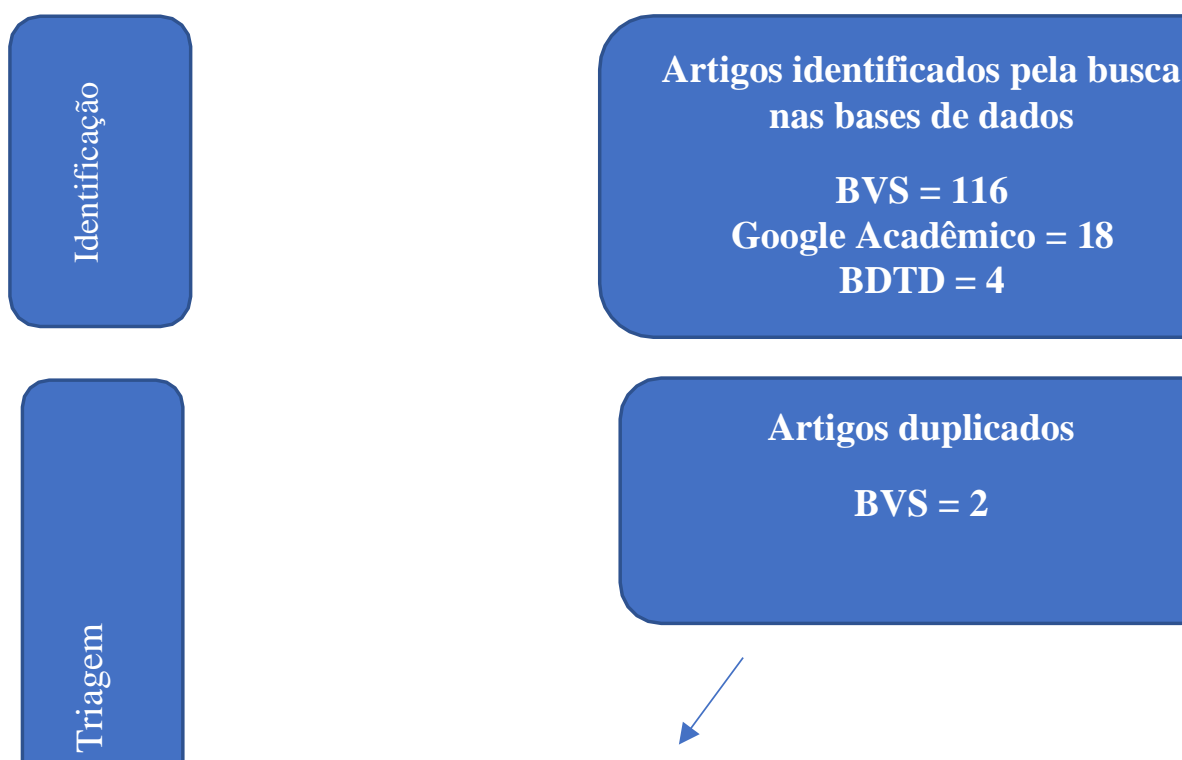

Artigos duplicados

BVS $=2$ 


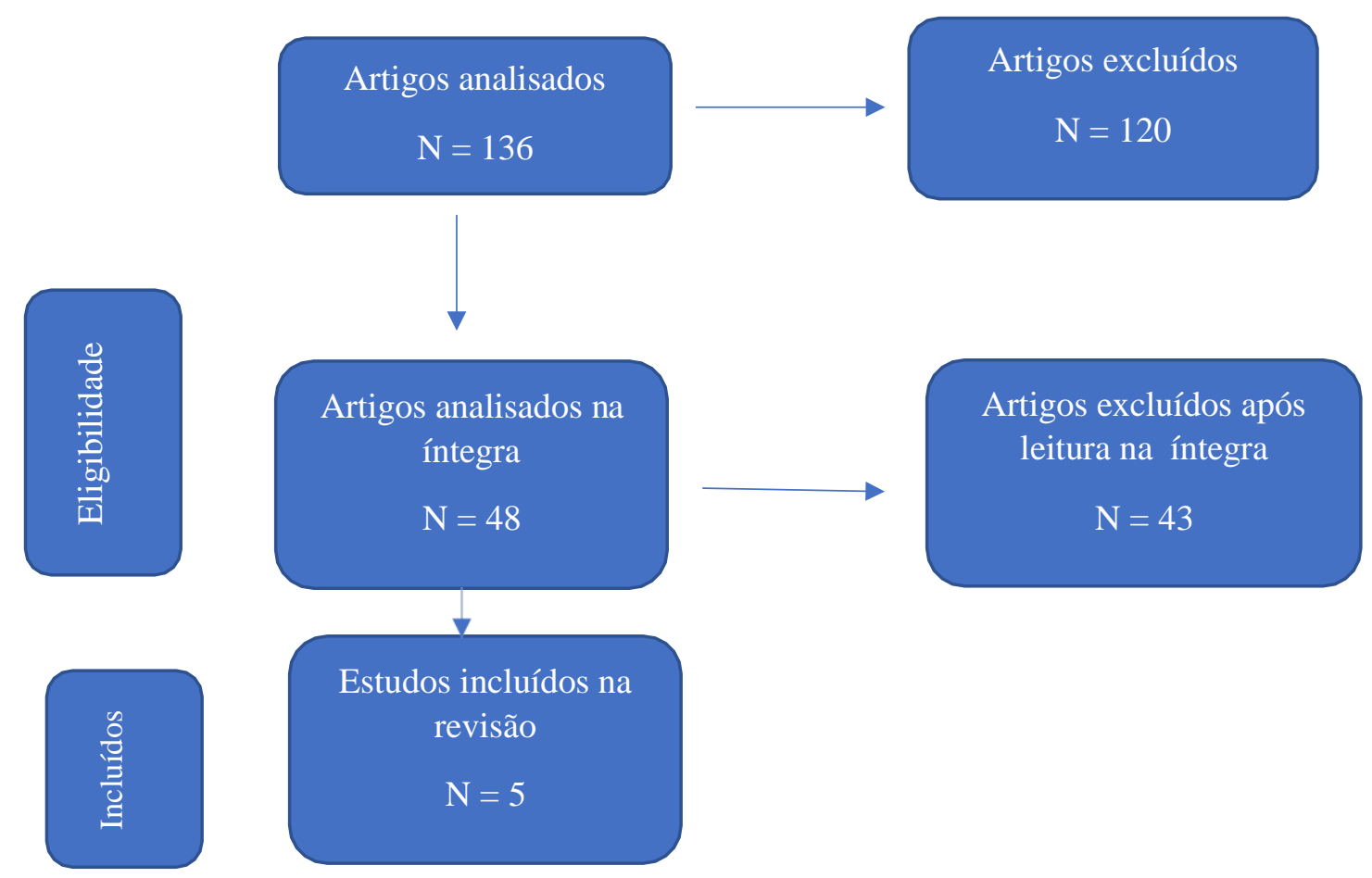

Fluxograma baseado no diagrama PRISMA.

Quadro 1. Publicações selecionadas nas bases de dados eletrônicas segundo suas características. Bahia, 2020

\begin{tabular}{|c|c|c|c|c|}
\hline $\begin{array}{l}\text { Título da } \\
\text { publicação }\end{array}$ & Periódico/Fonte & $\begin{array}{l}\text { Tipo de } \\
\text { publicação }\end{array}$ & Autores & Base de dados \\
\hline $\begin{array}{l}\text { R(existindo) ao caos: } \\
\text { A experiência de } \\
\text { Residentes no } \\
\text { Combate ao COVID- } \\
19 \text { na Atenção Básica }\end{array}$ & Fainor & $\begin{array}{l}\text { Relato de } \\
\text { experiência }\end{array}$ & $\begin{array}{l}\text { Matoso, } \\
\text { Silva e Silva }\end{array}$ & Google Acadêmico \\
\hline $\begin{array}{l}\text { A atuação do } \\
\text { cirurgião-dentista, } \\
\text { vinculado a um } \\
\text { programa de } \\
\text { residência } \\
\text { multiprofissional em } \\
\text { saúde, no combate a } \\
\text { COVID-19 na Atencão } \\
\text { Primária a Saúde: } \\
\text { Relato de experiencia }\end{array}$ & $\begin{array}{l}\text { Journal of } \\
\text { Management \& } \\
\text { Primary Health } \\
\text { Care }\end{array}$ & $\begin{array}{l}\text { Relato de } \\
\text { experiência }\end{array}$ & Santos et al. & Google Acadêmico \\
\hline $\begin{array}{l}\text { Atencão Básica a } \\
\text { Saúde no } \\
\text { enfrentamento a } \\
\text { COVID-19: } \\
\text { Perspectivas, desafios }\end{array}$ & $\begin{array}{l}\text { Revista } \\
\text { Interdisciplinar de } \\
\text { Pesquisa e } \\
\text { Inovação }\end{array}$ & $\begin{array}{l}\text { Relato de } \\
\text { experiência }\end{array}$ & Peixoto et al. & Google Acadêmico \\
\hline
\end{tabular}


e a experiencia de um

Programa de

Residência

multiprofissional em

saúde da Família

Building Telemedicine

Capacity for Trainess

During the Novel

Coronavirus Outbreak:

a Case Study and

Lessons Learned

Atenção Primária à

Saúde frente à

Enfermagem em

Relato de

Journal of

caso

General Internal

Medicine

foco

COVID-19: Relato de

experiência de um

Centro de Saúde

Fonte: Elaboração própria

\section{RESULTADOS E DISCUSSÕES}

Inicialmente foram identificadas cento e trinta e oito publicações sobre o tema. No entanto, priorizou-se a seleção de artigos que atendessem aos critérios de inclusão. Não obstante, aqueles que retrataram o processo de trabalho durante a pandemia gerada pelo novo Coronavírus não associados aos programas de residência voltados à atuação na Atenção Primária foram excluídos.

A base de dados que apresentou o maior número de artigos foi o Google Acadêmico, através da qual, foram incluídos cinco artigos correlacionados à proposta desta revisão. Acrescenta-se a este conjunto, um estudo de caso identificado no Pubmed/MEDLINE. Para completude da pesquisa, referências empregadas pelos autores dos artigos foram utilizadas, não sendo realizada pesquisa manual.

Todos os estudos incluídos na amostra foram publicados no ano de 2020 consoante período da pandemia causada pela Covid-19 conforme Quadro 1. Importante ressaltar que quatro publicações envolveram revistas brasileiras e foram divulgadas em língua portuguesa. Somente o estudo de caso fora publicado em inglês. Este envolveu residentes da Atenção Básica 
vinculados à Escola de Medicina Grossman da Universidade de Nova York. Por fim, nenhuma publicação de língua espanhola atendeu aos critérios de inclusão.

A maioria dos estudos qualitativos selecionados foram relatos de experiência (cerca de 90\% da amostra) sendo publicações brasileiras envolvendo Programas de Residência Multiprofissional que atuam na região Nordeste do país. Dentre as quais, duas publicações encenam no Estado da Bahia, duas no Rio Grande do Norte e uma no Estado de Sergipe. Apenas uma publicação vinculada à Programas de Residência Médica foi identificada.

Em todos os artigos incluídos na amostra houve o reconhecimento dos autores quanto às limitações dispostas na natureza dos estudos empregados. Limitações estas vinculadas a descrição de uma dada realidade que possa não refletir homogeneidade em relação às diferentes realidades globais ${ }^{20}$.

Três artigos distintos ${ }^{21,22,} 23$ refletiram sobre o papel da APS no enfrentamento da pandemia pelo novo Coronavírus e avaliaram de forma exitosa o engajamento da equipe de Residência Multiprofissional, no contexto sanitário vigente. Desta forma, pautaram a importância de publicações que compartilhem experiências de enfrentamento por diferentes programas de residência.

Neste novo contexto epidemiológico, para garantir atendimento seguro e de qualidade na atenção básica, com base nas considerações dos autores das publicações selecionadas, foi necessário planejamento e reorganização dos fluxos nos processos de trabalho. Para ratificar esta assertiva o relato de experiência das cirurgiãs-dentistas residentes ${ }^{22}$ descreveu as adaptações sugeridas pelo Ministério da Saúde no fluxo de atendimento de uma Unidade de Saúde da Família, no contexto da pandemia. Por conseguinte, ressaltaram o aprimoramento do cuidado aos pacientes sintomáticos respiratórios a partir de cursos promovidos pela esfera gestora federal assim como o enfretamento de subjetividades individuais associadas ao medo e insegurança. Adicionalmente três estudos ${ }^{21,24,23}$ trouxeram uma abordagem reflexiva sobre os 
desafios vivenciados pelas equipes da atenção básica diante da necessidade emergencial de mudança nos fluxos gerenciais e operacionais das ações e serviços em saúde. Foi trazido como exemplo: estrutura física incompatível com a nova demanda; escassez de EPI; precária higienização do ambiente de trabalho e a incompreensão dos usuários acerca das medidas sanitárias de prevenção ao coronavírus. À vista disso, os autores ressaltam a importância da APS no papel de promoção, prevenção e recuperação, avaliando a necessidade no direcionamento de recursos para aprimoramento na gestão do cuidado.

Não obstante, os desafios estruturais, não se limitaram à APS. Neste ínterim, as experiências relatadas em âmbito hospitalar, descritas através dos autores ${ }^{25,26}$ em pesquisas singulares, pontuaram os impactos que a carência de estruturas físicas ideais em enfermarias e unidades de terapia intensiva da rede pública poderiam causar no novo cenário epidemiológico, deixando em evidência possíveis fragilidades assistenciais passíveis de serem identificadas no Sistema Único de Saúde (SUS).

Por outro lado, objetivando a integralidade do cuidado na APS, o Ministério da Saúde regulamentou a telemedicina em atendimento excepcional no contexto pandêmico, através da Portaria n. 467, de 20 de março de $2020^{27}$. A literatura expõe benefícios importantes à população brasileira, através da orientação, monitoramento e encaminhamento para avaliação presencial oportuna dos sintomáticos respiratórios.

Destarte, autores ${ }^{28}$ através do seu relato de experiência como residentes da atenção básica, em concordância com as potencialidades desta nova ferramenta, analisaram que o telemonitoramento contribuiu de maneira ímpar na garantia de assistência e oferta de informação com maior segurança e eficácia, sem necessariamente haver comparecimento à USF, com vistas a evitar a contaminação e transmissão do novo coronavírus.

Consequentemente, no estado da Bahia, o aplicativo, denominado "Monitora-COVID" formulado pelas secretarias de Ciência, Tecnologia e Inovação (SECTI), juntamente com a 
Secretaria Estadual de Saúde (SESAB) em parceria com a Fundação Estatal Saúde da Família (FESF-SUS) despontou com objetivo de acompanhar o estado de saúde da população no sentido de identificar precocemente sinais e sintomas do novo coronavírus. Observou-se que o aplicativo favoreceu a classificação de risco de acordo com histórico clínico e sintomatologia atual relatados pelo usuário. De acordo com o risco calculado, o paciente recebe mensagens de texto e e-mails com orientações sanitárias de acordo com a classificação de risco assim como atendimento telefônico em até 24 horas por um médico, caso a sua atribuição de risco seja considerada elevada. Ademais, o monitoramento dos casos é realizado por todas as categorias profissionais da área da saúde, desde que este participe do treinamento inicial e pactuem ações continuadas de educação permanente ${ }^{29}$.

Dentre os artigos incluídos na amostra ${ }^{21,22,23,24,31}$ a abordagem sobre as novas formas de cuidado à distância está contida nos relatos de experiência ${ }^{23,31}$ através de um engajamento positivo através do teleatendimento na atenção básica para o rastreamento e monitoramento dos sintomáticos respiratórios de maneira eficaz.

Em contrapartida, o relato de experiência internacional dos residentes médicos inseridos em uma unidade na cidade de Nova York, discorre sobre as limitações e desafios operacionais na implementação da telemedicina, como nova ferramenta de cuidado, tendo em vista que não houve um treinamento prévio para mesma, resultando em insegurança na equipe para reprodução desta prática ${ }^{31}$.

No que tange às medidas de proteção e segurança no trabalho, dois relatos ${ }^{21,22}$ salientaram a escassez dos equipamentos de proteção individual e coletiva na APS, avaliando criticamente a exposição do profissional de saúde aos riscos físicos, químicos e biológicos além de possíveis repercussões negativas para o usuário na assistência à saúde. Entrementes, medidas de controle e racionamento de Equipamentos de Proteção Individual (EPI) foram descritas no contexto hospitalar do SUS $^{26}$. Os autores de ambas as pesquisas encerram suas discussões, 
salientando que a precarização do setor saúde tem gerado condições inadequadas de trabalho, fazendo-se necessário um melhor planejamento de recursos, objetivando o alcance da integralidade do cuidado.

Em relação a abordagem da saúde mental no contexto da pandemia, um estudo ${ }^{26}$ analisou criticamente a saúde do trabalhador, na qual, discutiu-se a vulnerabilidade dos profissionais diante da exposição contínua a fatores estressores provocados pela pandemia. $\mathrm{O}$ sofrimento psíquico exemplificado neste artigo, retratou o medo do contágio associado à possibilidade de contaminação dos seus familiares e aumento da sobrecarga de trabalho. Como considerações, os autores sugeriram a elaboração de um plano de cuidado para apoio psicológico ao trabalhador de saúde.

Por outro lado, no cotidiano do hospital de referência em infectologia da região nordeste autores $^{20}$ relataram experiência fundamentada no atendimento à pacientes internados com suspeita ou confirmação diagnóstica de COVID-19. À tempo destacou-se sofrimento e desgaste psicológico causados pelo processo de adoecimento e hospitalização destes pacientes. Sofrimento pela ausência física das redes de apoio familiar, medo de morrer, angústias acerca das exclusões sociais causadas pelos estigmas da doença e por fim, o medo de transmitir a doença foram algumas subjetividades relatadas pelos pacientes acolhidos. Nesse sentido, estratégias de enfrentamento através de ferramenta para interação virtual por chamadas de vídeo com familiares assim como apoio psicológico individual foram estabelecidas com o objetivo de minimizar o sofrimento destes pacientes. Neste interim, ressalta-se que esta revisão integrativa não identificou artigos que pautassem a saúde mental de usuários acolhidos na APS durante o período da pandemia em questão.

Por conseguinte, dois artigos ${ }^{32,23}$ retrataram o protagonismo da atenção básica, diante do seu espaço dinâmico e multiprofissional, onde foram executadas as ações de promoção, prevenção e recuperação, tendo capacidade elevada na redução de danos e agravos provocados 
pela COVID-19, além do encaminhamento qualificado e oportuno para outras redes de atenção à saúde. Consoante a essa discussão, foram relatadas ações desenvolvidas no serviço, com destaque para a descrição do empenho executado pelos agentes comunitários em saúde, na busca ativa e monitoramento dos sintomáticos respiratórios no território, assim como, orientação à população sobre medidas sanitárias de combate ao Coronavírus. Destaca-se que as ações de vigilância em saúde foram retratadas através da teleconsulta assim como acompanhamento longitudinal por meio das linhas de cuidado preexistentes na Atenção Básica.

Por fim, autores ${ }^{23,24}$ trouxeram que o país continua direcionando a maior parte das ações e recursos públicos para a atenção terciária com implementação de leitos hospitalares, unidades de terapia intensiva e hospitais de campanha, desconsiderando a importância da atenção básica na intensificação de medidas sanitárias e controle de epidemias no contexto histórico brasileiro.

\section{CONSIDERAÇÕES FINAIS}

Percebeu-se, a partir da análise dos artigos incluídos nesta pesquisa, uma convergência de opiniões sobre as contribuições dos programas de residência para o enfrentamento à COVID19 apesar de alterações no fluxo de atendimento e escassez de EPI.

Por fim, ao considerar as limitações dos relatos de experiência e o número reduzido de artigos encontrados, incentiva-se a produção de novas revisões com espaço temporal a fim de que seja acompanhada a produção dos Programas de Residência enquanto perdurar a pandemia gerada pelo novo Coronavírus.

\section{REFERÊNCIAS}

1. Pereira MD, Oliveira LC, Costa CFT, Bezerra CMO, Pereira MD, Santos CKA. A pandemia de COVID-19, o isolamento social, consequências na saúde mental e estratégias de enfrentamento: uma revisão integrativa. Res., Soc. Dev. [periódico na Internet] 2020 Jun 
[acesso 2020 Set 14]; 9(7): [cerca de 29 p.]. Disponível em: https://rsdjournal.org/index.php/rsd/article/view/4548/4043. DOI: 10.33448/rsd-v9i7.4548.

2. Schuchmann AZ, Schnorrenberger BL, Chiquetti ME, Gaiki RS, Raimann BW, Maeyama MA. Isolamento social vertical X Isolamento social horizontal: os dilemas sanitários e sociais no enfrentamento da pandemia de COVID-19. Braz. J. Hea. Rev [periódico na Internet] 2020 Abr [acesso 2020 Set 14]; 3(2): [cerca de 21 p.]. Disponível em: https://www.brazilianjournals.com/index.php/BJHR/article/view/9128/7738.DOI:10.34119/bj hrv3n2-185.

3. Schmidt B, Crepaldi MA, Bolze SDA, Neiva-Silva L, Demenech LM. Impactos na Saúde Mental e Intervenções Psicológicas Diante da Pandemia do Novo Coronavírus (COVID-19). SciELO Preprints [periódico na Internet]. 2020 Abr [acesso 2020 Set 15]; 1(1): [cerca de 27 p.]. Disponível em: https://www.researchgate.net/publication/340682336_Impactos_na_Saude_Mental_e_Interve ncoes_Psicologicas_Diante_da_Pandemia_do_Novo_Coronavirus_COVID19/link/5e98e97c299bf13079a1ce62/download. DOI: 10.1590/SciELOPreprints.58.

4. Lima LD, Pereira AMM, Machado CV. Crise, condicionantes e desafios de coordenação do Estado federativo brasileiro no contexto da COVID-19. Cad. Saúde Pública [periódico na Internet]. 2020 Jul [acesso 2020 Set 16];36(7): [cerca de 6 p.]. Disponível em: http://cadernos.ensp.fiocruz.br/static//arquivo/1678-4464-csp-36-07-e00185220.pdf. DOI: 10.1590/0102-311X00185220.

5. World Health Organization. WHO. Coronavirus disease (COVID-19) outbreak [Internet]. Geneva: World Health Organization; 2020 [cited 2020 Oct 16]. Available from: https://www.who.int/emergencies/ diseases/novel-coronavirus-2019.

6. Coelho FC, Lana RM, Cruz OG, Villela D, Bastos LS, Piontti AP et al. Assessing the potential impact of COVID-19 in Brazil: Mobility, Morbidity and the burden on the Health 
Care System. MedRxiv [serial on the Internet] 2020 March [cited 2020 Oct 16]: [about 17 p.]. Available from: https://www.medrxiv.org/content/10.1101/2020.03.19.20039131v2. DOI: https://doi.org/10.1101/2020.03.19.20039131.

7. Brasil. Ministério da Saúde. Agência Nacional de Vigilância Sanitária. Nota Técnica no 04/2020 GVIMS/GGTES/ANVISA. Orientações para serviços de saúde: medidas de prevenção e controle que devem ser adotadas durante a assistência aos casos suspeitos ou confirmados de infecção pelo novo coronavírus (sars-cov-2). Jan 2020. Acesso em 2020 Out 16. Disponível em: https://www20.anvisa.gov.br/segurancadopaciente/index.php/noticias/176-nota-tecnica-n04-2020-gvims-ggtes-anvisa-atualizada.

8. Brasil. Ministério da Saúde. Secretaria de Atenção Primária à Saúde. Protocolo de manejo clínico do coronavírus (Covid-19) na atenção primária à saúde. Abr 2020. Acesso em 2020 Out 18. Disponível em: https://saude.rs.gov.br/upload/arquivos/202004/14140606-4-msprotocolomanejo-aps-ver07abril.pdf.

9. Brasil. Ministério da Saúde. Nota Técnica nº 09/2020-CGSB/DESF/SAPS/MS. COVID-19 e atendimento odontológico no SUS. Mar 2020. Acesso em 2020 Out 18. Disponível em: https://www.abo.org.br/uploads/files/2020/03/covid-19-atendimento-odontologico-no-sus.pdf. 10. Brasil. Ministério da Saúde. Procedimento Operacional Padronizado. Equipamento de proteção individual e Segurança no trabalho para profissionais de saúde da APS no atendimento às pessoas com suspeita ou infecção pelo novo coronavírus (covid-19). Acesso em 2020 Out 19. Disponível em: https://renastonline.ensp.fiocruz.br/sites/default/files/arquivos/recursos/procedimento_operaci onal_padrao_epi.pdf.

11. Brasil. Ministério da Saúde. Portaria no 580 de 27 de março de 2020. Dispõe sobre a Ação Estratégica "O Brasil Conta Comigo - Residentes na área de Saúde", para o enfrentamento à pandemia do coronavírus (COVID-19). Diário Oficial da União 2020; 30 mar. Acesso em 2020 
Out 22. Disponível em: https://www.in.gov.br/en/web/dou/-/portaria-n-580-de-27-de-marcode-2020-250191376.

12. Brasil. Casa Civil. Lei 11.129 de 30 de junho de 2005. Art. 13. Institui a Residência em Área Profissional da Saúde, definida como modalidade de ensino de pós-graduação lato sensu, voltada para a educação em serviço e destinada às categorias profissionais que integram a área de saúde, excetuada a médica. Diário Oficial da União 2005; 30 jun. Acesso em 2020 Out 22. Disponível em: http://www.planalto.gov.br/ccivil_03/_Ato2004-2006/2005/Lei/L11129.htm. 13. Brasil. Ministério da Saúde. Portaria nº 1.143 de 07 de julho de 2005. Apoia programas de residência médica em medicina de família e comunidade (PRM-MFC), por meio do Programa de Bolsas para a Educação pelo Trabalho, do Ministério da Saúde. Diário Oficial da União 2020; 07 jul. Acesso em 2020 Out 22. Disponível em: https://www.in.gov.br/en/web/dou/-/portaria-n-467-de-20-de-marco-de-2020-249312996.

14. Brasil. Casa Civil. Lei 12.871 de 22 de outubro de 2013. Art. 13. Institui o Programa Mais

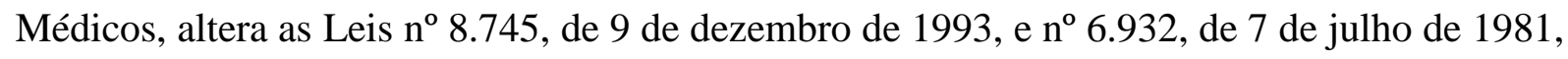
e dá outras providências. Diário Oficial da União 2013; 22 out. Acesso em 2020 Out 23. Disponível em: http://www.planalto.gov.br/ccivil_03/_ato20112014/2013/lei/112871.htm\#: :text=Institui\%20o\%20Programa\%20Mais\%20M\%C3\%A9dicos ,1981\%2C\%20e\%20d\%C3\%A1\%20outras\%20provid\%C3\%AAncias.\&text=VIII\%20\%2D\% 20estimular\%20a\%20realiza\%C3\%A7\%C3\%A3o\%20de\%20pesquisas\%20aplicadas\%20ao\% 20SUS.

15. Rosa SD, Vasconcelos EMA. Residência Multiprofissional em Saúde e Políticas de Formação Profissional. Seminário Internacional de Educação Superior 2014 - Formação e Conhecimento; 2014; Sorocaba-SP: Uniso; 2014. 
16. Alves J, Carvalho P. Importância do Programa de Residência Multiprofissional em Saúde da Família da Universidade Federal de Pernambuco na formação dos profissionais da área de saúde. EFDeportes.com, Revista Digital [periódico na Internet]. 2014 Jan [acesso 2020 Out 23]; 28(2): [cerca de 7 p.]. Disponível em: https://www.efdeportes.com/efd188/formacao-dosprofissionais-de-saude.htm.

17. Silva JC, Contim D, Ohl RIB, Chavaglia SRR, Amaral EMS. Percepção dos residentes sobre sua atuação no programa de residência multiprofissional. Acta Paul Enferm [periódico na Internet]. 2015 Out-Nov [acesso 2020 Out 23]; (188): [cerca de 7 p.]. Disponível em: https://www.scielo.br/scielo.php?script=sci_arttext\&pid=S0103-

21002015000200132\&lng=pt\&tlng=pt. DOI: http://dx.doi.org/10.1590/1982-0194201500023.

18. Whittemore R, Knafl K. The integrative review: updated methodology. J Adv Nurs [serial on the Internet]. 2005 Dec [cited 2020 Out 23]; 52(5): [about 7 p.]. Available from: https://pubmed.ncbi.nlm.nih.gov/16268861/. DOI: 10.1111/j.1365-2648.2005.03621.x.

19. Prisma [homepage na Internet]. Transparent Reporting of Systematic Reviews and MetaAnalyses. Acesso em 2020 Out 23. Disponível em: http://prismastatement.org/prismastatement/flowdiagram.aspx.

20. Silva KCL, Lima MEG. A inserção de duas psicólogas residentes em tempos da Covid-19. Cadernos Esp. Ceará [periódico na Internet]. 2020 Jul [acesso 2020 Out 23]; 14(1): [cerca de 5 p.]. Disponível em: https://cadernos.esp.ce.gov.br/index.php/cadernos/article/view/316/219. 21. Matoso LML, Silva AAB, Silva FA. R(existindo) ao caos: a experiência de residentes no combate ao COVID-19 na atenção básica. C\&D-Revista Eletrônica da FAINOR [periódico na Internet]. 2020 Set-Dez [acesso 2020 Out 24]; 13(3): [cerca de 16 p.]. Disponível em: http://srv02.fainor.com.br/revista/index.php/memorias/article/view/1114/563.

DOI:10.11602/1984-4271.2020.13.3.14. 
22. Santos JSX, Silva AS, Carvalho LA, Soares JO, Lopes SPA, Moreira MBA. A atuação do cirurgião-dentista, vinculado a um programa de residência multiprofissional em saúde, no combate à COVID-19 na Atenção Primária à Saúde: relato de experiência. J Manag Prim Health Care [periódico na Internet]. 2020 Jul [acesso 2020 Out 24]; 12: [cerca de 16 p.]. Disponível em: $\quad$ https://www.jmphc.com.br/jmphc/article/view/993. DOI: https://doi.org/10.14295/jmphc.v12.993.

23. Peixoto MVS, Carvalho S, Silva RJS, Silva FA, Silva WB. Atenção básica à saúde no enfrentamento à COVID-19: perspectivas, desafios e a experiência de um programa de residência multiprofissional em saúde da família. RevIPI [periódico na Internet]. 2020 Ago [acesso 2020 Out 24]; 7(2): [cerca de 12 p.]. Disponível em: https://seer.ufs.br/index.php/revipi/article/view/14214.

24. Rios AFM, Lira LSSP, Reis IM, Silva GA. Atenção primária à saúde frente à COVID-19 em um centro de saúde. Enferm. foco (Brasília) [periódico na Internet]. 2020 Ago [acesso 2020 Out24]; 11(1): [cerca de 6 p.]. Disponível em: https://pesquisa.bvsalud.org/portal/resource/pt/biblio-1116877.

25. Silva ANM, Santos AP. Atuação do fisioterapeuta residente em infectologia no contexto da COVID-19: possibilidades e desafios. Cadernos Esp. Ceará [periódico na Internet]. 2020 Jul [acesso 2020 Out 24]; 14(1): [cerca de 5 p.]. Disponível em: https://cadernos.esp.ce.gov.br/index.php/cadernos/article/view/380/230.

26. Freire RMS, Batista GS, Carvalho TA, Silva DS, Faustino TN, Merces MC. Profissional residente no enfrentamento da COVID-19: relato de experiência no contexto da enfermagem intensiva. Revista Enfermagem Brasil. Enferm Bras [periódico na Internet]. 2020; 19(4Supl): S13-S20. Acesso 2020 Out $25 . \quad$ Disponível em: http://portalatlanticaeditora.com.br/index.php/enfermagembrasil/article/view/4299. DOI: http://dx.doi.org/10.33233/eb.v19i4.4299. 
27. Brasil. Ministério da Saúde. Portaria no 467 de 20 de março de 2020. Dispõe, em caráter excepcional e temporário, sobre as ações de Telemedicina, com o objetivo de regulamentar e operacionalizar as medidas de enfrentamento da emergência de saúde pública de importância internacional previstas no art. $3^{\circ}$ da Lei $\mathrm{n}^{\circ} 13.979$, de 6 de fevereiro de 2020, decorrente da epidemia de COVID-19. Diário Oficial da União 2020; 23 mar. Acesso em 2020 Out 26. Disponível em: https://www.in.gov.br/en/web/dou/-/portaria-n-467-de-20-de-marco-de-2020249312996.

28. Rodrigues AP, Felipe CR, Lima DB, Costa LRO, Fernandes PF, Silva RPP, et al. Telemonitoramento como estratégia de cuidado longitudinal a grupos prioritários em -VitóriaES. APS. [periódico na Internet]. 2020 Jun [acesso 2020 Out 26];2(2) [cerca de 8 p.]. Disponível em: $\quad<h t t p s: / / a p s e m r e v i s t a . o r g / a p s / a r t i c l e / v i e w / 100>$. DOI: https://doi.org/10.14295/aps.v2i2.100.

29. Bahia. Governo do Estado [homepage na Internet]. Aplicativo Monitora Covid-19 está disponível na Apple e Play Store. Acesso em 2020 Out 26. Disponível em: http://www.seplan.ba.gov.br/2020/05/1458/Aplicativo-Monitora-Covid-19-esta-disponivelna-Apple-e-Play-Store.html.

30. Felisberto LCC, Giovannini PE, Diógenes ICF, Lucas Pontes Nunes Carlos LPN, Lins LFTS. O caminho se faz ao caminhar: novas perspectivas da educação médica no contexto da pandemia. Rev. bras. educ. med. [periódico na Internet]. 2020 Out; 44(1Supl): S01-S08. [acesso 2020 Out 27]. Disponível em: https://www.scielo.br/pdf/rbem/v44s1/1981-5271-rbem-44-s1e156.pdf. DOI: https://doi.org/10.1590/1981-5271v44.supl.1-20200422.

31. Lawrence KMPH, Hanley J, Adams J, Sartori DJ, Greene R, Zabar S. Building Telemedicine Capacity for Trainees During the Novel Coronavirus Outbreak: a Case Study and Lessons Learned. J Gen Intern Med. 2005 Jul [cited 2020 Oct 27]; 35(9): [about 5 p.]. Available 
from: https://www.ncbi.nlm.nih.gov/pmc/articles/PMC7343380/. DOI: 10.1007/s11606-02005979-9.

32. Lucena JF, Sena JGB. Residência integrada multiprofissional em saúde e a pandemia COVID-19: um relato de experiência. REAS/EJCH [periódico na Internet]. 2020 Set [acesso 2020 Oct 27]; 12(9): [cerca de 6 p.]. Disponível em: https://www.acervomais.com.br/index.php/saude/article/view/4964. DOI: https://doi.org/10.25248/reas.e4964.2020. 